\title{
Endoscopic Ultrasound Guided Choledocho-Enterostomy by Using Lumen Apposing Metal Stent in Patients with Failed Endoscopic Retrograde Cholangiopancreatography: A Literature Review
}

\author{
Deepanshu Jain ${ }^{a}$ Manan Shah $^{b}$ Upen Patel $^{b}$ Abhinav Sharma ${ }^{b}$ \\ Shashideep Singhal ${ }^{\mathrm{b}}$ \\ ${ }^{a}$ Division of Gastroenterology and Hepatology, Department of Digestive Diseases and Transplantation, Einstein \\ Healthcare Network, Philadelphia, PA, USA; ${ }^{b}$ Division of Gastroenterology, Hepatology and Nutrition, University of \\ Texas Health Science Center at Houston, Houston, TX, USA
}

\section{Keywords}

Endoscopic ultrasound - Bile drainage - Obstructive jaundice · Lumen apposing metal stent

\begin{abstract}
Background: Endoscopic retrograde cholangiopancreatography (ERCP) has been the cornerstone in the management of malignant jaundice to provide symptomatic relief and/or to allow chemotherapy. Difficult biliary cannulation or the presence of concomitant duodenal obstruction may lead to its failure. Method: An extensive English literature search was done via PubMed and Google Scholar to identify 13 peer-reviewed original articles. Results: Of 92 patients, 98.9\% (91/92) successfully underwent EUS-guided biliary drainage by placement of a lumen-apposing metal stent (LAMS) across extrahepatic biliary tree and small bowel. Although technically challenging, a similar technical success was noted for patients with limited dilation of extrahepatic biliary tree (less than $1 \mathrm{~cm}$ ) and altered gastrointestinal anat-
\end{abstract}

(c) 2018 S. Karger AG, Basel

E-Mail karger@karger.com www.karger.com/dig omy (Roux-en-Y, Whipple, or Billroth II). Of 91 patients, $98.9 \%$ (90/91) had clinical success. Procedure complications included perforation $(2 / 92 ; 2.2 \%)$ and bleeding $(1 / 92 ; 1.1 \%)$. Stent complications included obstruction $(7 / 91 ; 7.7 \%)$, and migration $(1 / 91 ; 1.1 \%)$ with all responding to endoscopic debridement of food or tumor, placement of plastic pigtail stent through the LAMS, or replacement with a new stent. No procedure-related mortality was noted. Conclusion: In the hands of an expert, EUS-guided biliary drainage with the use of LAMS is an efficacious and safe option for patients with distal malignant biliary stricture, not amenable to ERCP.

(c) 2018 S. Karger AG, Basel

\section{Introduction}

As per cancer statistics, there will be 53,670 new pancreatic cancer cases in 2017 in the United States of America [1]. A majority of pancreatic cancers occur in the head of the pancreas [1]. The 5-year relative survival rate is 
approximately $7 \%$ [2]. The most widely recognized reason for a malignant distal biliary obstruction is pancreatic head cancer. Palliative biliary drainage diminishes symptoms and improves symptoms for these patients with advanced cancer who are not candidates for surgery [3]. Endoscopic retrograde cholangiopancreatography (ERCP) is the most accepted and widely used method for bile drainage in patients with obstructive jaundice [4]. However, ERCP may not be feasible if cannulation fails or if the scope cannot be passed to reach the papilla due to proximal digestive tract obstruction. Percutaneous transhepatic biliary drainage (PTBD) is an alternative method for biliary drainage if ERCP fails. However, PTBD can be associated with complications like bile leak, bleeding, bile peritonitis, bowel perforation, pneumothorax, secondary infections, and catheter dislodgement in upto $30 \%$ of the patients $[4,5]$. An external drainage tube can be cumbersome and uncomfortable for the patient [6]. Ascites is one of the absolute contraindications for PTBD [7].

EUS-guided bile drainage (EUS-BD) via choledochoduodenostomy (CD) was first introduced in 2001 [8]. Since then it has been performed using traditional plastic or metal stents originally designed for trans-papillary drainage by ERCP. However, stent occlusion, bile leak, and subsequent bile peritonitis is often seen with the use of pigtail plastic stents [9]. Metal stents, on the other hand, due to their larger size and rigid nature are less often associated with occlusion and bile leak [9]. But they came at the cost of higher incidence of stent migration and small bowel wall trauma due to their tubular shape and lack of anti-migration design [10]. In addition, among patients with concomitant duodenal and biliary obstruction, stents placed across EUS-CD for biliary drainage interfere with the placement of luminal duodenal stents and vice versa.

EUS-BD by using lumen-apposing metal stent (LAMS) is an innovative and a relatively new technique. LAMS was originally designed to provide solid anchorage between nonadherent luminal structures. The stent is built from braided nitinol wire to grant adaptability and is completely covered with silicone to counteract tissue ingrowth, prevent tract leakage, and allow removability. Its flanges are intended to disperse pressure uniformly on the luminal wall. The AXIOS stent (Xlumena, Inc., Mountain View, CA, USA) comprises double-walled flanges perpendicular to the lumen, which hold the tissue walls in apposition to prevent stent migration $[11,12]$.

In this review article, we have summarized case reports, case series, and multicenter prospective studies to evaluate the technique, composite efficacy, safety, and feasibility of LAMS in the management of bile drainage for patients in whom primary treatment modality like ERCP is technically nonfeasible.

\section{Materials and Methods}

An extensive English language literature search was done till August 2017 using Pubmed and Google scholar to identify the peer-reviewed original and review articles using keywords - Endoscopic ultrasound; Bile drainage; Obstructive jaundice; Lumen apposing metal stent. Only articles about studies that included human subjects were selected. The references to relevant studies were manually searched to identify additional relevant studies. The search yielded case reports [13-22], case series [23,24], and a prospective multicenter study [25]. The indication for procedure, prior interventions, patient characteristics, LAMS specifics, procedure details, technical and clinical success rates, complications with their management, and limitations of each study were reviewed and have been summarized in Table 1 .

\section{Results}

Thirteen original articles were considered appropriate to be included in this review article [13-25]. In our review, we included 10 case reports - 2 from Spain $[16,20]$, 3 from Japan [17-19], 4 from the United States [14, 15, $21,22]$, and 1 from Italy [13]. In addition, we included 2 case series - one of which comes from Italy [23] while the other one was a multicenter study from Germany, Denmark, Italy, and Spain [24]. A prospective multicenter study from Japan and Hong-Kong was also included in our review [25].

\section{Discussion}

\section{Etiology of Biliary Obstruction}

A majority of patients (90/92) had malignant biliary obstruction [13-17, 20-25]. Pancreatic cancer was the most common type, whereas duodenal, ampullary, gastric, cholangiocarcinoma, sarcoma, and metastatic cancer (renal, colon, breast, or uterine) attributed to the rest [13-17, 20-25]. One patient had benign biliary obstruction secondary to stenosis at the site of choledochojejunostomy from prior whipple surgery [18] and another patient presented with recurrent cholangitis due to intrahepatic bile duct stones who had surgical cholecystoduodenostomy for congenital biliary atresia as a child [19]. 


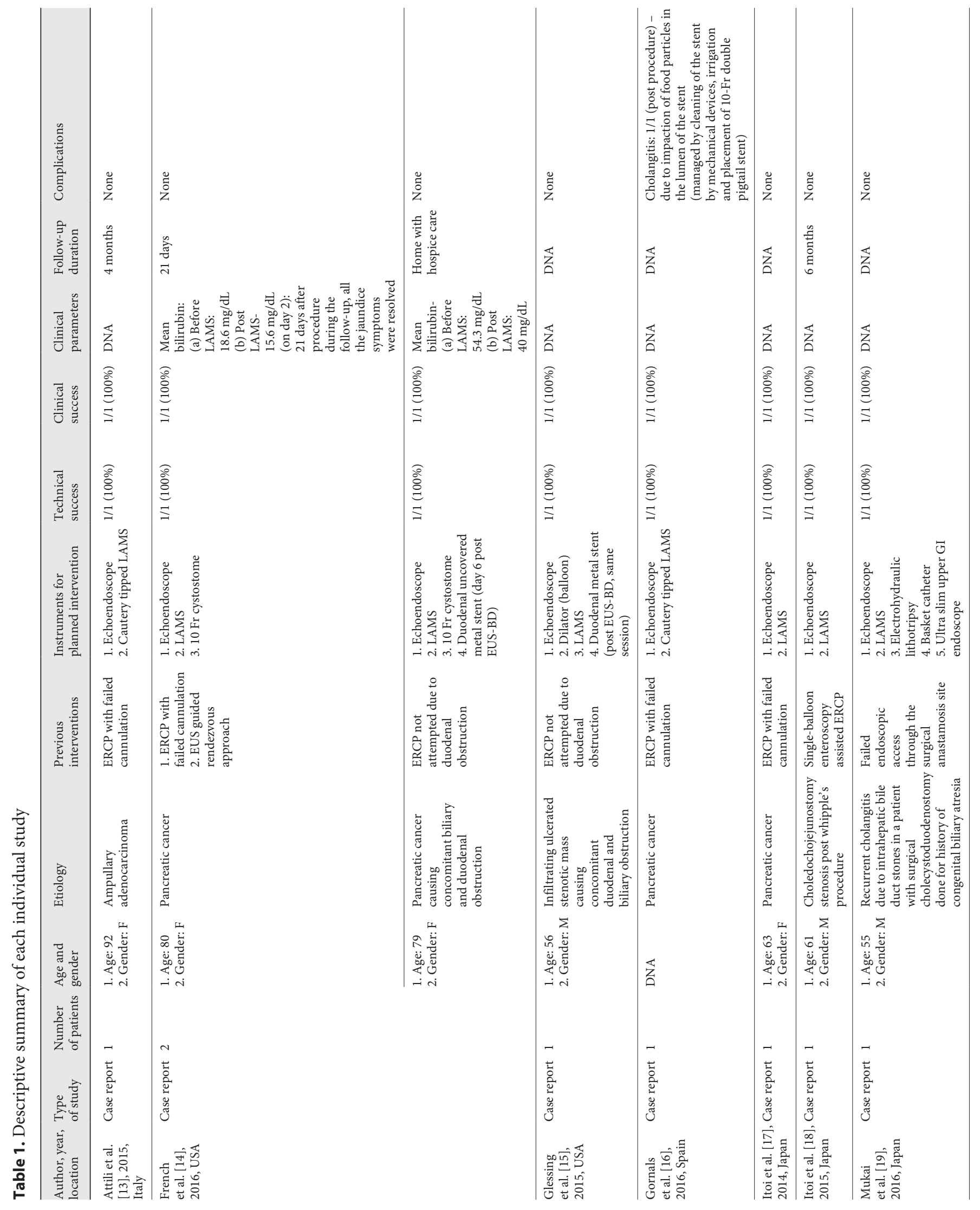




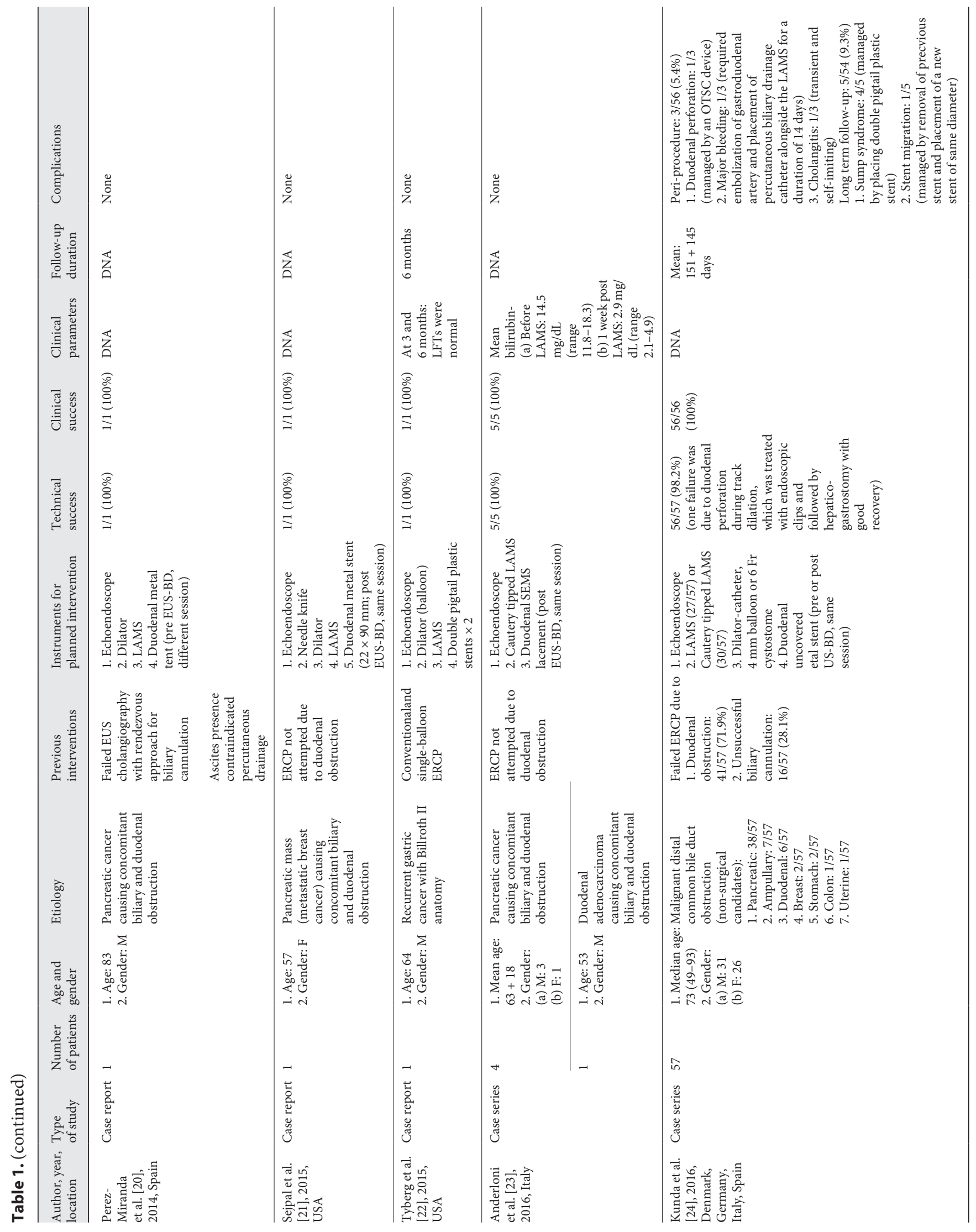




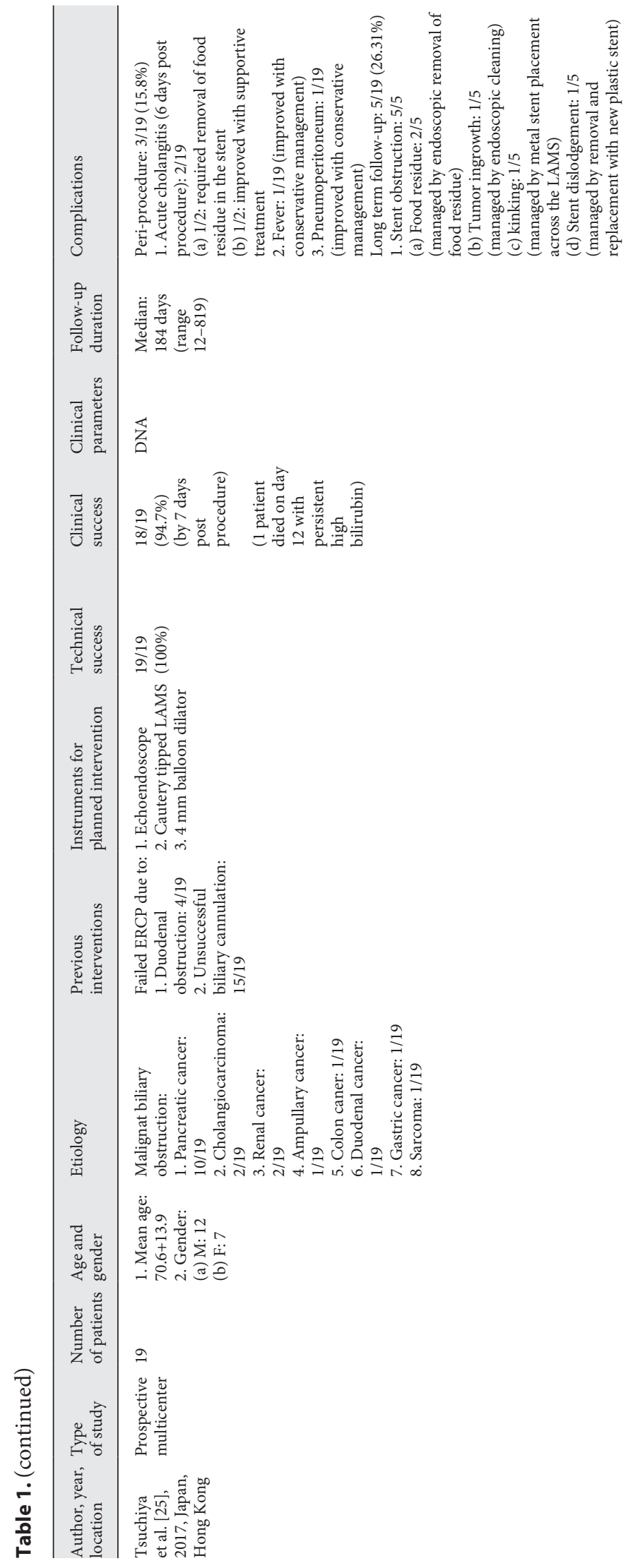

\section{Prior Interventions}

The indication for EUS-guided BD essentially comes from failure of primary therapeutic modality - ERCP. For each patient there was either a failure to achieve biliary cannulation $[13,14,16,17,20,24,25]$ or the ERCP in itself was technically challenging due to duodenal obstruction $[14,15,21,23-25]$ or altered GI anatomy from prior surgery $[18,19,22]$. Even single-balloon enteroscopy-assisted ERCP $[18,22]$ and biliary cannulation via rendezvous approach $[14,20]$ failed in 4 cases. Majority of the patients had preferentially selected EUS-BD via CD over PTBD approach. In a few, the concomitant presence of ascites also contraindicated the PTBD [20, 24, 25].

\section{Procedure Characteristics}

\section{Technique}

Across all studies, a therapeutic linear echoendoscope was used to guide the creation of a fistulous communication between extra hepatic biliary tree and duodenum $[13-17,19-21,23-25]$ or jejunum $[18,22]$. Once the dilated extra hepatic biliary was identified on the EUS, a 19 $\mathrm{G}$ needle was used to puncture it $[14,20-22,24,25]$. The position was then confirmed either by free outward flow of bile or by contrast infusion and its visualization under the fluoroscopy. Needle was followed by passage of a guide wire, which was either 0.025 inch [25] or 0.035 inch $[14,21,24]$ thick. This was followed by a dilator, which was a balloon, a catheter, or a cystostome depending on the endoscopist's preference and availability $[14,15,20$ $22,24]$. The size of the dilator depended on the pre-procedure dilation of the biliary tree and the intended size of the LAMS to be placed. Newer studies have shown the use of an electro-cautery tipped delivery system for LAMS, which allowed direct placement of the LAMS without necessitating the need of wire placement or dilation of track before the stent placement [13, 16, 23-25]. Sejpal et al. [21] in their only case used a needle knife to puncture the duodenal wall before dilating it to allow LAMS placement. Once within the biliary tree, the proximal flange was deployed first under EUS guidance, followed by minimal pulling of stent to allow adequate apposition of the walls of bile duct and small bowel and then finally the distal flange is deployed either under fluoroscopic guidance or direct visualization. Once deployed, dilation of the stent lumen is performed as needed by using a $4-8$ $\mathrm{mm}$ balloon dilator [25]. Tyberg et al. [22] placed 2 pigtail plastic stents in addition to LAMS to separate it from the opposite duct wall. 
Six studies describe placement of duodenal metal stent to treat concomitant duodenal stricture $[15,23,24]$. The duodenal stent was placed on the same or different session either before or after LAMS placement by EUS-BD. Also, one author describes its placement overlapping LAMS [15], while another author avoided overlapping [23]. LAMS due to its short-length and design does not hinder the placement of a concomitant duodenal stent and vice versa.

\section{LAMS Specifics}

In our review, various-sized LAMSs were used across different studies. The size of the stent to be used was decided by the endoscopist based on the size of the bile duct [13-25]. Tsuchiya et al. [25] reported that no statistically significant difference was found for clinical success rate and stent patency rate between 6 and $8 \mathrm{~mm}$ diameter LAMS. Individual stent specifics for each study are summarized in the Table 2.

\section{Duration}

Only 3 studies reported the length of the procedure. The median procedure time for Kunda et al. [24] and Tsuchiya et al. [25] was 22.4 and $16.2 \pm 7.3$ min respectively. Anderloni et al. [23] had a mean procedure time of $19 \pm$ _0.8 min (range 18-20 min).

\section{Technical Success and Failure}

Technical success was defined as the ability to perform EUS-BD by successfully deploying LAMS across the bile duct into the small bowel. All but one study had $100 \%$ technical success rate. Out of 92 patients from 13 individual studies, technical success was achieved in 91 patients (98.9\%). Kunda et al. [24] reported failure in one patient due to duodenal perforation while attempting to dilate the track to insert LAMS. The patient was successfully managed with endoscopic clips and had EUS-guided hepatico-gastrostomy with uneventful recovery [24].

The size of the extra-hepatic biliary tree plays a key role in determining the technical feasibility for LAMS placement. Although the majority of authors mention the presence of a dilated biliary tree, only 4 studies actually reported the true size $[13,14,24,25]$. Seventy-nine patients from these 4 studies had a mean/median bile duct size of more than $15 \mathrm{~mm}[13,14,24,25]$. Kunda et al. [24] reported successful EUS-BD via LAMS in 3 patients with the bile duct size less than $10 \mathrm{~mm}$. The author did report that it was challenging to find a dilated part of bile duct and then to release the distal flange of LAMS within it. The author concluded that new users should perform a procedure only if bile duct is at least $15 \mathrm{~mm}$ in diameter [24].

Other factors that may potentially challenge the endoscopist performing this procedure include inability to position the echoendoscope in the bulb of the duodenum due to intrinsic luminal mass, extrinsic compression, gastric outlet obstruction, or altered gastrointestinal tract anatomy due to prior surgeries like-whipple, Billroth II, Roux-en-Y gastric bypass, and so on. Itoi et al. [18] and Tyberg et al. [22] reported success in EUS-BD via LAMS placement in 2 patients with altered GI anatomy. Six studies had patients with concomitant duodenal and biliary obstruction $[14,15,20,21,23,24]$. Depending on the anatomy and location of obstruction, few authors placed metal stents first to allow EUS-BD via LAMS placement $[20,24]$. In others, duodenal stents were placed post EUSBD via LAMS $[14,15,21,23,24]$. In the majority of the study patients, duodenal stents were placed in the same session $[15,21,23,24]$ except for 2 patients $[14,20]$.

The site of obstruction in the biliary tree may also undermine the technical success of this procedure. Proximal biliary strictures like hilum strictures anatomically leaves a shorter portion of the biliary tree, which is dilated and extrahepatic in location, thus making it technically challenging to spot the ideal location for LAMS deployment under EUS guidance. Tsuchiya et al. [25] excluded such patients from their study cohort.

\section{Clinical Success and Failure}

Clinical success was defined as the improvement in obstructive jaundice and/or cholestatic laboratory parameters. Non-procedure-related mortality was not considered a clinical failure, as the aim of EUS-BD via LAMS was palliative in a majority of the studies [13-17, 20-25] except two $[18,19]$. Out of 91 patients from 13 individual studies with successful EUS-BD via LAMS, clinical success was reported in 90 patients (98.9\%). All studies had a clinical success rate of $100 \%$ [13-24] except one [25]. Tsuchiya et al. [25] reported the improvement of obstructive jaundice in 18 out of 19 patients (94.7\%). One patient continued to have worsening of liver function tests (LFTs) despite technically successful procedure. The same patient expired 12 days after the procedure from worsening pancreatic cancer. In the case reported by Mukai et al. [19], the EUS-CD via LAMS placement allowed ultra slim upper GI endoscope to reach intrahepatic ducts with successful electrohydraulic lithotripsy followed by basket catheter method of extraction of stones. Kunda et al. [24] 


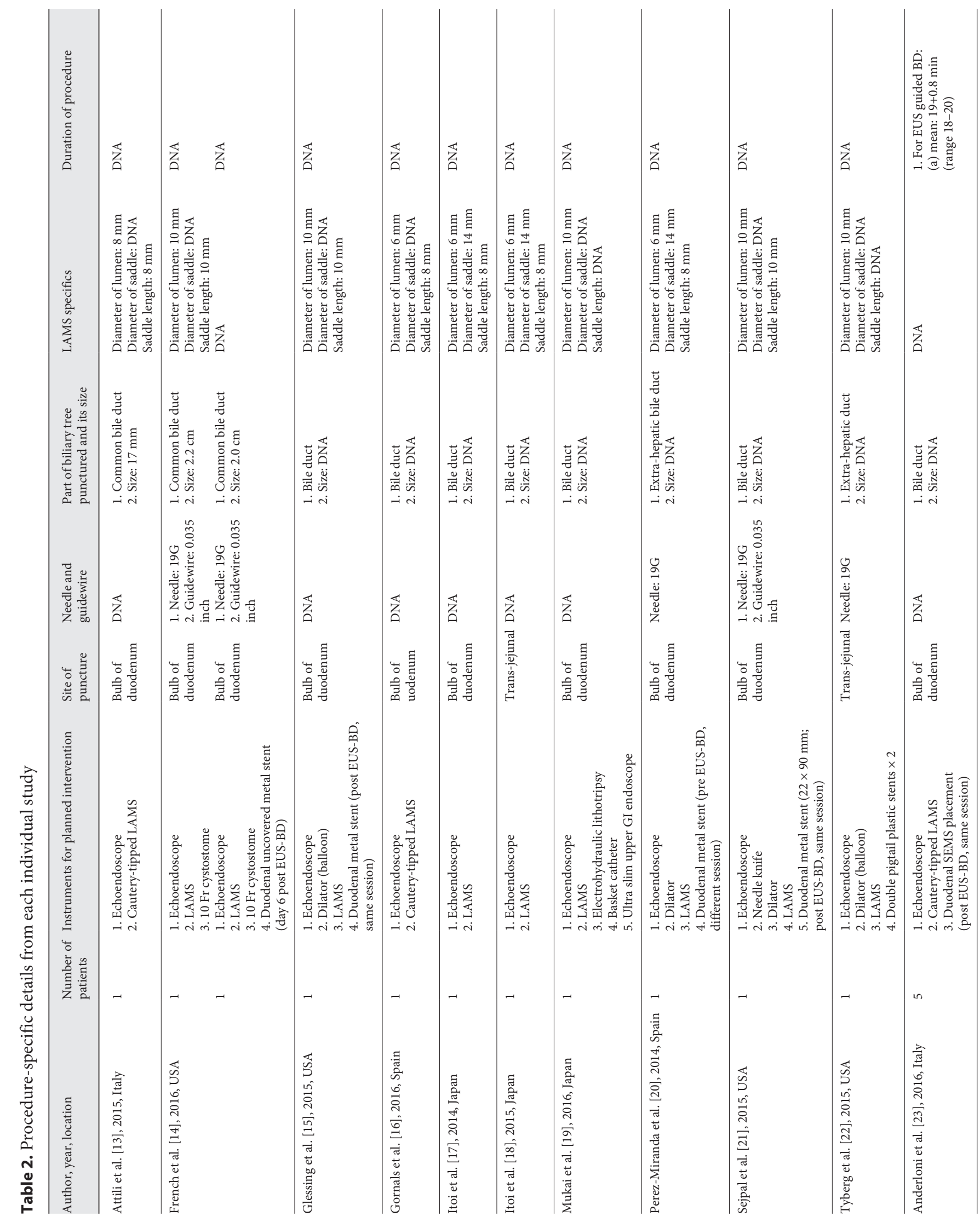




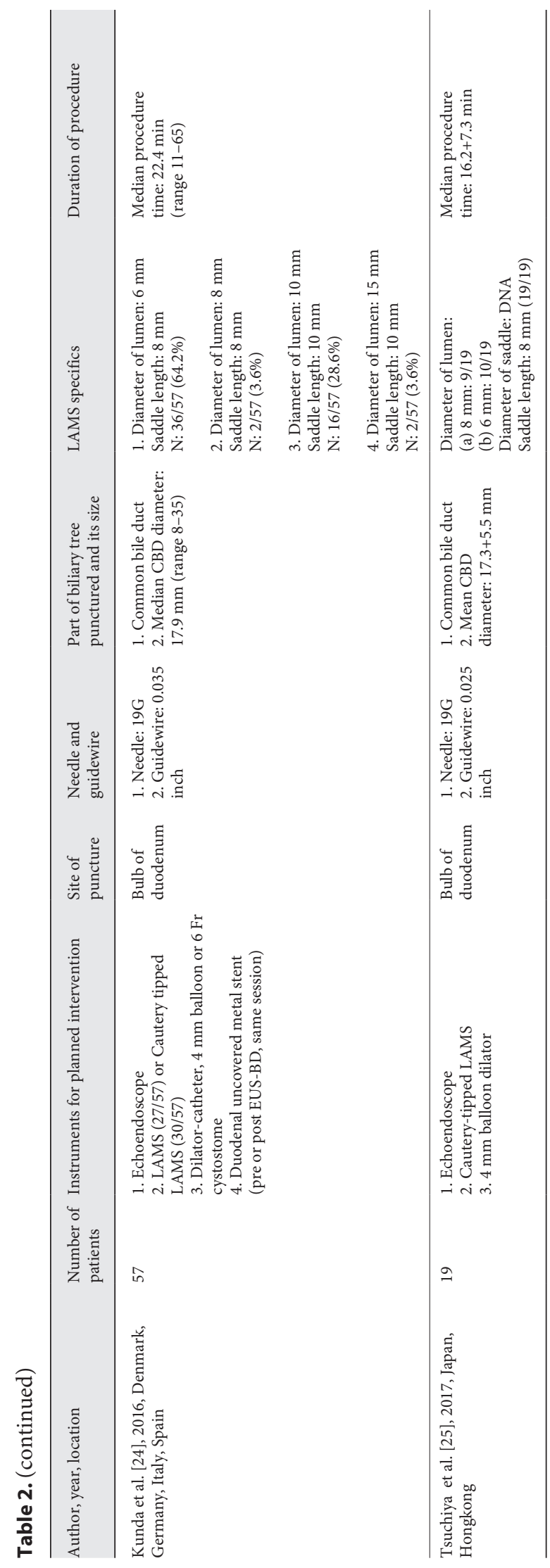

reported 2 deaths within 4 days post-LAMS placement despite effective biliary drainage as noted by an improvement in the serum bilirubin level. The cause of death was attributed to multiorgan failure, which was ongoing even before EUS-BD [24]. On study-specific follow-up interval, author reported either complete resolution of abnormal LFTs [22] or a significant improvement [14, 23]. The details from each study have been summarized in Table 1 .

\section{Follow-Up}

As majority of procedures were for palliative indication, no specific follow-up was reported for patients once an improvement in symptoms and/or LFTs was documented after the procedure. Of studies with available information, Kunda et al. [24] and Tsuchiya et al. [25] reported the longest mean follow-up duration of $151 \pm 145$ days and median follow-up of 184 days (range 12-819) respectively.

\section{Complications}

Out of 13 studies, 10 studies reported no complications [13-15, 17-20, 22, 23]. Three studies reported complications like stent occlusion leading to cholangitis, stent migration, bleeding, small bowel perforation, and sump syndrome $[16,24,25]$. For the sake of discussion, we have categorized them into early (periprocedure) and late complications.

\section{Periprocedure Complications}

These included adverse events that were observed during the intra-procedure or up to 1-week post procedure.

Perforation or Pneumoperitoneum

In the study cohort of 92 patients, 2 perforations [24] and 1 pneumoperitoneum [25] were reported. One perforation led to technical failure and its management and outcome has been described above under the technical failure heading [24]. Other perforation happened from the tip of the EUS scope during evaluation of the site before LAMS placement. This defect was successfully treated with over-thescope clip and was followed by EUS-BD via LAMS at an alternative location with technical and clinical success [24]. Tsuchiya et al. [25] reported a case of pneumoperitoneum, which was managed conservatively with good outcome.

\section{Bleeding}

Of 92 patients, one patient developed severe acute arterial bleed in duodenum post insertion of cautery-tipped LAMS, which was treated with embolization of gastro- 
duodenal artery [24]. The author also placed a percutaneous biliary drainage catheter alongside LAMS for 2 weeks following which it was removed.

\section{Cholangitis or Fever}

A total of 5 patients developed fever (1/5) [25] or cholangitis $(4 / 5)[16,24,25]$ post EUS-BD via LAMS placement. One patient with fever [25] and 2 patients with cholangitis $[24,25]$ improved with supportive care alone. Other 2 patients were found to have food residue within the stent and improved after mechanical debridement of stent $[16,25]$. Gornals et al. [16] in addition to irrigation and mechanical removal of food debris placed a $10 \mathrm{Fr}$ double pigtail plastic stent within LAMS to prevent the recurrence of a similar episode in future.

\section{Stent Obstruction}

Tsuchiya et al. [25] reported a patient who developed stent obstruction from food debris on day 6 post procedure and required endoscopic cleaning.

\section{Late Complications}

These adverse events were reported beyond 1 week from procedure day.

\section{Stent Obstruction}

Out of 92 patients, 4 patients developed stent obstruction on long-term follow-up [25]. The etiology varied from food debris (day 193; 1/5), tumor ingrowth (1/5), kinking (day 15; 1/5), or stent dislodgement (day 328; 1/5) [25]. All of them required repeat endoscopic intervention: mechanical cleaning of residual food, debridement of tumor, deployment of metal stent across the LAMS, or removal of LAMS with placement of new plastic stent across the fistula [25].

\section{Stent Migration}

Kunda et al. [24] reported one patient with stent migration, which required replacement with a new LAMS of same diameter across the previously created fistulous tract.

\section{Sump Syndrome}

Of the study cohort, 4 patients were found to have fluctuant abnormalities in the LFTs on follow-up [24]. ERCP revealed the presence of sludge in the distal biliary tree but no obvious obstruction or food residue. This was successfully managed by transient placement of double pigtail plastic stent across the LAMS for the duration of 2 weeks.

EUS-Guided Biliary Drainage via LAMS

\section{Training}

To date, there is no evidence-based structured training for this technique. So far, only few experts in the field have performed the procedure. To be proficient, expertise is required at 2 steps - EUS-guided access of the biliary tree and LAMS deployment. Experience and comfort level of the mentor, baseline skill set of trainee, and volume of cases are likely to play critical role in determining the learning curve of this technique for naïve advanced fellows-in-training.

\section{Conclusion}

Although the experience is limited, EUS-guided choledocho-enterostomy by using LAMS for patients with distal malignant biliary stricture who are unamenable to ERCP has a high technical and clinical success rate with a low complication rate. The risk of publication bias cannot be excluded and hence the results of this review should be interpreted with caution and procedure should be performed only by experienced endoscopists with adequate surgical backup.

\section{Disclosure Statement}

None of the authors has any conflicts of interest or financial relationship with the company that produces or distributes the device described in the review article.

\section{Funding Source} article.

No grant or funding source was received for writing this review

\section{References}

1 Siegel RL, Miller KD, Jemal A: Cancer Statistics, 2017. CA Cancer J Clin 2017;67:7-30.

$\checkmark 2$ Miller KD, Siegel RL, Lin CC, et al: Cancer treatment and survivorship statistics, 2016. CA Cancer J Clin 2016;66:271-289.

3 Abraham NS, Barkun JS, Barkun AN: Palliation of malignant biliary obstruction: a prospective trial examining impact on quality of life. Gastrointest Endosc 2002;56:835-841.

4 Artifon EL, Loureiro JF, Baron TH, et al: Surgery or EUS-guided choledochoduodenostomy for malignant distal biliary obstruction after ERCP failure. Endosc Ultrasound 2015; 4:235-243.

5 Akhan O, Akinci D, Ozmen MN: Percutaneous cholecystostomy. Eur J Radiol 2002;43: 229-236. 
6 Kedia P, Tyberg A, Kumta NA, et al: EUS-directed transgastric ERCP for Roux-en-Y gastric bypass anatomy: a minimally invasive approach. Gastrointest Endosc 2015;82:560565.

-7 Ring EJ, Kerlan RK Jr: Interventional biliary radiology. AJR Am J Roentgenol 1984;142: 31-34.

8 Giovannini M, Moutardier V, Pesenti C, et al: Endoscopic ultrasound-guided bilioduodenal anastomosis: a new technique for biliary drainage. Endoscopy 2001;33:898-900.

9 Park DH, Jang JW, Lee SS, et al: EUS-guided biliary drainage with transluminal stenting after failed ERCP: predictors of adverse events and long-term results. Gastrointest Endosc 2011;74:1276-1284.

10 Prachayakul V, Aswakul P: Endoscopic ultrasound-guided biliary drainage as an alternative to percutaneous drainage and surgical bypass. World J Gastrointest Endosc 2015;7: 37-44.

11 Itoi T, Binmoeller KF, Shah J, et al: Clinical evaluation of a novel lumen-apposing metal stent for endosonography-guided pancreatic pseudocyst and gallbladder drainage (with videos). Gastrointest Endosc 2012;75:870876.

12 Binmoeller KF, Shah J: A novel lumen-apposing stent for transluminal drainage of nonadherent extraintestinal fluid collections. Endoscopy 2011;43:337-342.

13 Attili F, Rimbas M, Galasso D, et al: Fluoroless endoscopic ultrasound-guided biliary drainage after failed ERCP with a novel lumen-ap- posing metal stent mounted on a cauterytipped delivery system. Endoscopy 2015; 47(suppl 1):E619-E620.

14 French JB, Coe AW, Pawa R: Endoscopic ultrasound-guided choledochoduodenostomy with a lumen-apposing, self-expandable fully covered metal stent for palliative biliary drainage. Clin J Gastroenterol 2016;9: 79-85.

15 Glessing BR, Mallery S, Freeman ML, et al: EUS-guided choledochoduodenostomy with a lumen-apposing metal stent before duodenal stent placement for malignant biliary and duodenal obstruction. Gastrointest Endosc 2015;81:1019-1020.

16 Gornals JB, Consiglieri CF, Bergamino MA: Double pigtail for preventing ascending cholangitis after endoscopic ultrasonographyguided choledochoduodenostomy with $\mathrm{lu}$ men-apposing metal stent. Dig Endosc 2016; 28:100.

17 Itoi T, Binmoeller KF: EUS-guided choledochoduodenostomy by using a biflanged lumen-apposing metal stent. Gastrointest Endosc 2014;79:715.

18 Itoi T, Ikeuchi N, Tonozuka R, et al: EUSguided choledochojejunostomy with a lumen-apposing metal stent in a post-Whipple patient. Gastrointest Endosc 2015;81:12591260.

19 Mukai S, Itoi T, Tsuchiya T, et al: EUS-guided intrahepatic bile duct stone extraction via choledochoduodenostomy created by a lumen-apposing metal stent. Gastrointest Endosc 2016;83:832-833.
20 Perez-Miranda M, De la Serna Higuera C, Gil-Simon P, et al: EUS-guided choledochoduodenostomy with lumen-apposing metal stent after failed rendezvous in synchronous malignant biliary and gastric outlet obstruction (with video). Gastrointest Endosc 2014; 80:342; discussion 343-344.

21 Sejpal DV, Trindade AJ, Vamadevan AS: Lumen-apposing metal stent placement for biliary drainage may be preferable in the setting of duodenal obstruction when dual stenting is performed. Gastrointest Endosc 2015;82:170.

22 Tyberg A, Karia K, Zerbo S, et al: Endoscopic ultrasound-guided choledochojejunostomy with a lumen-apposing metal stent: a shortcut for biliary drainage. Endoscopy 2015;47(suppl 1 UCTN):E342-E343.

23 Anderloni A, Buda A, Carrara S, et al: Singlesession double-stent placement in concomitant malignant biliary and duodenal obstruction with a cautery-tipped lumen apposing metal stent. Endoscopy 2016;48(suppl 1): E321-E322.

24 Kunda R, Perez-Miranda M, Will U, et al: EUSguided choledochoduodenostomy for malignant distal biliary obstruction using a lumenapposing fully covered metal stent after failed ERCP. Surg Endosc 2016;30:5002-5008.

25 Tsuchiya T, Bun Teoh AY, Itoi T, et al: Long-term outcomes of EUS-guided choledochoduodenostomy using a lumen-apposing metal stent for malignant distal biliary obstruction: a prospective multicenter study. Gastrointest endosc 2017;pii:S00165107(17)32199-5. 